TRANSACTIONS OF THE

AMERICAN MATHEMATICAL SOCIETY

Volume 364, Number 2, February 2012, Pages 767-784

S 0002-9947(2011)05320-1

Article electronically published on September 15, 2011

\title{
INTEGRAL POINTS AND VOJTA'S CONJECTURE ON RATIONAL SURFACES
}

\author{
YU YASUFUKU
}

\begin{abstract}
Using an inequality by Corvaja and Zannier about gcd's of polynomials in $S$-units, we verify Vojta's conjecture (with respect to integral points) for rational surfaces and triangular divisors. This amounts to a gcd inequality for integral points on $\mathbb{G}_{m}^{2}$. The argument in the proof is generalized to give conditions under which Vojta's conjecture on a variety implies Vojta's conjecture on its blowup.
\end{abstract}

\section{INTRODUCTION}

Building on the work of Charles Osgood 11] which connected Roth's theorem with Nevanlinna theory, Paul Vojta [15] formulated a precise dictionary between value distribution theory and diophantine approximations. Using this dictionary, he translated Griffiths' conjecture in complex analysis to a powerful height inequality for rational points of smooth varieties [15, Main Conjecture 3.4.3]. Vojta's conjecture tells us how diophantine approximation on a variety is controlled by its canonical divisor, and it has profound consequences in both arithmetic and geometry: its known cases include Schmidt's subspace theorem (Schmidt [13] and Schlickewei [12]) and two of Faltings' major results (Mordell conjecture 44 for curves and Lang's conjecture [5] for abelian varieties), and its unsolved cases include the Bombieri-Lang conjecture and the $a b c$ conjecture. As indicated by these examples, Vojta's conjecture is very difficult to prove in general.

As the geometry of the blowup is closely related to the original variety, one would expect Vojta's conjecture on the variety and on its blowup to be similar. In fact, Vojta's conjecture on the blowup implies Vojta's conjecture on the base, and moreover, these statements do not differ by much [15, Example 3.5.4]. However, proving Vojta's conjecture on the blowup is still often nontrivial even if the conjecture is known for the base: for blowups of abelian varieties, the only known case is the blowup of $E \times E$, where $E$ is a rank-1 elliptic curve (McKinnon 10]). Moreover, Vojta's conjecture on blowups often implies new arithmetic results, including interesting greatest-common-divisor (gcd) inequalities and properties of elliptic divisibility sequences, as Silverman [14] has explored.

Received by the editors June 19, 2009 and, in revised form, November 26, 2009, January 26, 2010, and February 13, 2010.

2010 Mathematics Subject Classification. Primary 11J97, 14G40; Secondary 14J26.

Key words and phrases. Vojta's conjecture, rational surfaces, integral points, blowups, multiplicative groups, greatest common divisors, Farey sequences.

This work was supported in part by NSF VIGRE grant number DMS-9977372. 
The main result of this paper (Theorem 1), to be made precise in the next subsection, deals with Vojta's conjecture on multiple blowups of $\mathbb{P}^{2}$. We prove that Vojta's height inequality holds on multiple blowups of $\mathbb{P}^{2}$ with a divisor "coming from" a triangle, when we look at the "integral points". As we will see, this gives us a gcd inequality for integral points on $\mathbb{G}_{m}^{2}$. The result for the case of the first blowup of $\mathbb{P}^{2}$ was previously observed by Silverman [14 to be equivalent to the highly nontrivial inequality of the gcd of polynomial expressions of $S$-units, proved by Corvaja and Zannier [3. To prove the multiple blowup case in general, we again use [3] together with some inductive arguments. Some care is necessary in the induction, since the theorem for the $n$-th blowup does not generally imply the theorem for the $(n+1)$-st blowup. Instead, we analyze the geometry to determine where the result of [3] is applicable and where inductive arguments work. The restriction to "integral points" is used for the induction part of the argument, as well as for applying [3].

We note that if we start from a similar setup as above but change the divisor from a triangle or remove the restriction to "integral points", the cases of Vojta's conjecture we can treat become small. In fact, if we change the divisor from a triangle to an irreducible cubic or to the union of an irreducible conic and a line, then the first blowup case is already unknown. As for the integrality condition, even if we weaken it a little by asking for integrality with respect to two lines rather than three (this amounts to looking at integral points of $\mathbb{G}_{m} \times \mathbb{A}^{1}$ ), the first blowup case seems very difficult. More detailed analysis of these cases, including what we can prove and the obstacles that we face, will be discussed at the end of the paper.

The paper is organized as follows. In the next subsection, we present the precise formulation of Vojta's conjecture and the main result of the paper (Theorem 1). Since Vojta's conjecture on the blowup implies the conjecture on the base, this theorem can be reformulated for rational surfaces (Corollary 4). In Section 2, we compute height functions on blowups. In particular, Propositions 8 and 9 and Example 10 (involving Farey sequences) will be essential. We also include a very general height computation on blowups (Proposition [5), as this does not seem to have appeared before. Section 3 contains the proof of Theorem 1 . In the final section, we discuss both provable and unsolved extensions of Theorem 1]. Proposition 16] generalizes the inductive argument in the proof of Theorem 1 to obtain geometric/arithmetic conditions under which Vojta's conjecture on a variety implies Vojta's conjecture on its blowup. We then end the paper by stating some explicit gcd inequalities, which are open problems related to the main theorem.

1.1. Precise statement. Let $k$ be a number field, let $M_{k}$ be the set of absolute values up to equivalence, and for each $v \in M_{k}$, let $|\cdot|_{v}$ be the absolute value in the class of $v$ which is the $\frac{\left[k_{v}: \mathbb{Q}_{v}\right]}{[k: \mathbb{Q}]}$-th power of the normalized absolute value on $\mathbb{Q}$. Additively, we write $v(x)=-\log |x|_{v}$, so the product formula becomes $\sum_{v \in M_{k}} v(x)=0$ for $x \in k^{*}$. Let $S$ be a finite set of places which includes $M_{\infty}$, the set of archimedean ones. We will use $\mathcal{O}$ for the ring of integers, $\mathcal{O}_{S}$ for the $S$-integers, and $\mathcal{O}_{S}^{*}$ for the $S$-units. Let $X$ be a projective variety over $k$. A variety is always assumed to be irreducible in this paper. For any Cartier divisor $D$ on $X$, let $h_{D}$ be a Weil height. $D$ corresponds to a line bundle $\mathscr{L}$ and a meromorphic section $s$, and a locally $M_{k}$-bounded metric $\|\cdot\|$ on $\mathscr{L}(D)$ gives us a local height function $\lambda_{D}(P, v)$ by the formula $\|s(P)\|_{v}$. With our normalization, $\sum_{v} \lambda_{D}(P, v)=h_{D}(P)$ up to a bounded function [1, 8, 9. When $D$ is effective, we define a set of $S$-integral points 
with respect to $D$ via local heights, as the set of points for which $\sum_{v \notin S} \lambda_{D}(-, v)$ is bounded by a constant [15. This definition agrees with the usual notion when $D$ is ample.

Vojta's conjecture [15, Main Conjecture 3.4.3]. Let $X$ be a smooth projective variety over $k, K$ a canonical divisor of $X, A$ an ample divisor, and $D$ a normal crossings divisor (by definition $D \geq 0$ and $D$ has no multiple components). Fix height functions $\lambda_{D}(-, v), h_{K}$, and $h_{A}$. Given $\epsilon>0$, there exists a Zariski-closed $Z_{\epsilon} \subsetneq X$ and a bounded function $O(1)$ such that

$$
\sum_{v \in S} \lambda_{D}(P, v)+h_{K}(P)<\epsilon h_{A}(P)+O(1)
$$

for $P \in X(k)$ not on $Z_{\epsilon}$.

To state the main result of this paper, let $X^{(0)}=\mathbb{P}^{2}$ and define $X^{(n+1)}$ inductively as the blowup of $X^{(n)}$ at a point $P_{n}$, with $E^{(n+1)}$ as the exceptional divisor. Let $K^{(n)}$ be a canonical divisor of $X^{(n)}$. For $i<n$, define $E^{(i,(i+1))}$ as the strict transform of $E^{(i)}$ to $X^{(i+1)}$, define $E^{(i,(i+2))}$ as the strict transform of $E^{(i,(i+1))}$ to $X^{(i+2)}$, and continue the process until we define $E^{(i, n)}$. We note that $E^{(i, n)}$ is an irreducible divisor which is isomorphic to $\mathbb{P}^{1}$. Let $\pi_{j i}: X^{(j)} \longrightarrow X^{(i)}$ denote the composition of blowups. Outside the set $B=\left\{\pi_{10}\left(E^{(1)}\right), \ldots, \pi_{n 0}\left(E^{(n)}\right)\right\}$ of at most $n$ points of $\mathbb{P}^{2}, \pi_{n 0}$ is an isomorphism, and we will often make this identification.

Theorem 1. Let $L=L_{1}+L_{2}+L_{3}$ be three lines in $\mathbb{P}^{2}$ defined over $\bar{k}$ with no triple intersections. Let $\widetilde{L}$ be the strict transform of $L$ to $X^{(n)}$. Let $D$ be a divisor on $X^{(n)}$ of the form $\widetilde{L}+\sum \chi_{i} E^{(i, n)}$, where $\chi_{i}$ is either 0 or 1 . Then Vojta's inequality on $X^{(n)}$ for the divisor $D$ holds for $S$-integral points $I$ in $\mathbb{P}^{2} \backslash|L|$. Namely, given $\epsilon>0$ and an ample divisor $A$ on $X^{(n)}$, there exists a Zariski-closed $Z \subsetneq X^{(n)}$ and a bounded function $O(1)$ such that

$$
\sum_{v \in S} \lambda_{D}(P, v)+h_{K^{(n)}}(P)<\epsilon h_{A}(P)+O(1)
$$

for $P$ in $\pi_{n 0}^{-1}(I \backslash B) \backslash Z$.

Example 2. If the first blowup occurs at a point not on $L$, by [7, exercise II.8.5], $K^{(1)}=-\widetilde{L}+E^{(1)}$. In this case, $D=\widetilde{L}+E^{(1)}$ is normal crossings, and

$$
\sum_{v \notin S} \lambda_{\widetilde{L}}(P)=\sum_{v \notin S} \lambda_{\pi^{*} L}(P) \leq O(1) \quad \text { for } P \in I .
$$

Therefore, (11) is equivalent to

$$
h_{E^{(1)}}(P)<\epsilon h_{A}(P)+O(1) \quad \text { for } P \in I .
$$

Example 3. If the first blowup occurs at an intersection point of $L$ and the second blowup occurs at a point on $E^{(1)}$ not on the strict transform of $L$, then $K^{(1)}=-\widetilde{L}-$ $E^{(1)}$ and $K^{(2)}=-\widetilde{L}-E^{(1,2)}$. For normal crossings divisor $D=\widetilde{L}+E^{(1,2)}+E^{(2)}$, (11) says

$$
-\sum_{v \notin S} \lambda_{\widetilde{L}}(P, v)-\sum_{v \notin S} \lambda_{E^{(1,2)}}(P, v)+\sum_{v \in S} \lambda_{E^{(2)}}(P, v)<\epsilon h_{A}(P)+O(1) .
$$


Since we can put all $E^{(i, n)}$ into $Z$, the full Vojta's conjecture involves evaluation of height inequality for points in $\mathbb{P}^{2}-B-|L|$ (upon identification via $\pi_{n 0}$ ). So the notion of integrality used in Theorem 1 is not as strange as it may seem at first. Moreover, when $D$ contains four or more components, integral points of $X^{(n)} \backslash|D|$ often lie in a proper Zariski-closed set [2, so Vojta-type statements would be vacuous. We will see that $I$ is the set of integral points on $\mathbb{G}_{m}^{2}$ (points with $S$-unit coordinates), and hence $I$ is Zariski-dense.

As noted earlier, this result for the first blowup of $\mathbb{P}^{2}$ is equivalent to the result by Corvaja and Zannier [3], which is proved by an ingenious application of Schmidt's subspace theorem in an arbitrarily high number of variables. In contrast, Vojta's conjecture on $\mathbb{P}^{2}$ for union of lines is equivalent to Schmidt's subspace theorem in three variables. Also, we only know the first blowup case for integral points, rather than for rational points as in the case of $\mathbb{P}^{2}$. Thus, the essentials of Theorem 1 are twofold: first, no further restriction of points is necessary to prove the conjecture for subsequent blowups; and second, the proof uses [3] rather than a yet-different application of Schmidt's subspace theorem.

The proof of Theorem 11 has several parts. We will show that some $h_{E^{(i, n)}}$ are "small" (that is, bounded above by $\epsilon h_{A}$ ) using explicit height computations of Section 2, the result in 3], and some geometric arguments. Otherwise $h_{E^{(i, n)}}$ is "big", but then such an $E^{(i, n)}$ satisfies a special geometric condition ("type two" divisor as defined later) which is reflected in $K^{(n)}$. Using these characterizations, we will do separate induction for each $E^{(i, n)}$. As an aside, Farey sequences will make their appearance in local heights of blowups which are toric. We end this section by reformulating the main result for rational surfaces.

Corollary 4. Let $X$ be a smooth rational surface, let $D$ be a normal crossings divisor, and let $K$ be a canonical divisor. Let $\pi_{1}: X^{\prime} \longrightarrow \mathbb{P}^{2}$ and $\pi_{2}: X^{\prime} \longrightarrow X$ be birational morphisms. Let $B$ be the set of points of $\mathbb{P}^{2}$ over which $X \leftarrow X^{\prime} \rightarrow \mathbb{P}^{2}$ is not an isomorphism. If $\pi_{1}\left(\pi_{2}^{*}(D)\right)$ as a set is a triangle $|L|$ plus some points in $\mathbb{P}^{2}$, then given $\epsilon>0$, there exists a Zariski-closed $Z_{\epsilon} \subsetneq \mathbb{P}^{2}$ and a bounded function $O(1)$ such that

$$
\sum_{v \in S} \lambda_{D}(P, v)+h_{K}(P)<\epsilon h_{A}(P)+O(1)
$$

for all S-integral points of $\mathbb{P}^{2} \backslash|L|$ which lie outside of $Z_{\epsilon} \cup B$.

Proof. If $\pi_{2}$ is a composition of $m$ blowups and if we let $F^{(j, m)}$ be the strict transforms to $X^{\prime}$ of various exceptional divisors, then by induction, the divisor $D^{\prime}=$ $\widetilde{D}+F^{(1, m)}+\cdots+F^{(m)}$ on $X^{\prime}$ is normal crossings. Since $\pi_{2}^{*}(D)=\widetilde{D}+\sum_{j} a_{j} F^{(j, m)}$ for $a_{j} \geq 1$, the images under $\pi_{1}$ of $\pi_{2}^{*}(D)$ and $D^{\prime}$ are the same. By assumption, $D^{\prime}$ must be of the form $\widetilde{L}+\sum_{i} \chi_{i} E^{(i, n)}$ if $X^{\prime}$ is an $n$-fold blowup of $\mathbb{P}^{2}$. By Theorem 1 , Vojta's conjecture holds for $\left(X^{\prime}, D^{\prime}\right)$ for integral points of $\mathbb{P}^{2} \backslash L$. Hence, the same holds for $(X, D)$ by Example 3.5.4 of [15].

\section{Heights ON BLOWUPS}

In this section, we will prove a general formula for heights on blowups with respect to exceptional divisors. In the next subsection, we will specialize this formula for multiple blowups of $\mathbb{P}^{2}$. For more details, see [17. 
Proposition 5. Let $X$ be a smooth projective variety over $k$, and let $\mathscr{I}$ be an ideal sheaf which defines a subscheme of codimension at least 2. Let $\left\{U_{i}\right\}$ be a finite cover of $X$ by open affines, and let $\left.\mathscr{I}\right|_{U_{i}}=\left(f_{i 1}, \ldots, f_{i m_{i}}\right)$. Then a local height function on the blowup $\mathrm{Bl}_{\mathscr{I}} X$ of $X$ along $\mathscr{I}$ with respect to the exceptional divisor $E$ is given by

$$
\lambda_{E}(P, v)=\max \left(0, \max _{\left\{i: P \in U_{i}\right\}} \min _{m} v\left(f_{i m}(P)\right)\right) .
$$

In this formula, $P$ outside of $E$ is identified isomorphically via the blowup map to the point in $X$ outside $\operatorname{Supp} \mathscr{I}$.

Remark. A local height is big at points which are $v$-adically close to the divisor. In the case of blowups, this happens when the image via the blowup map is close to $\operatorname{Supp} \mathscr{I}$. That is, for some $i,\left|f_{i 1}(P)\right|_{v}, \ldots,\left|f_{i m_{i}}(P)\right|_{v}$ should be all close to zero, so (4) makes sense.

Proof. It suffices to check this on one set of generators. Moreover, an embedding of $X$ into $\mathbb{P}^{N}$ induces an embedding of $\mathrm{Bl}_{\mathscr{I}} X$ into $\mathrm{Bl}_{\mathscr{I}} \mathbb{P}^{N}$, with exceptional divisors corresponding to each other. So we can assume $X=\mathbb{P}^{N}$, and $f_{1}, \ldots, f_{r} \in k\left[x_{0}, \ldots, x_{N}\right]$ homogeneous of degree $d$ which generate $\mathscr{I}$. Over $x_{i} \neq 0$, the blowup is given by

$$
\text { Proj } k\left[\frac{x_{0}}{x_{i}}, \ldots, \frac{x_{n}}{x_{i}}\right]\left[\alpha_{1}: \cdots: \alpha_{r}\right] / I,
$$

where $I$ contains relations of the form $\frac{f_{j}}{x_{i}^{d}} \alpha_{j^{\prime}}-\frac{f_{j^{\prime}}}{x_{i}^{d}} \alpha_{j}$ among others. Here, by $\operatorname{Proj} R\left[\alpha_{1}: \cdots: \alpha_{r}\right] / I$, we mean the Proj of the graded ring where elements of ring $R$ are given degree $0, \alpha_{j}$ 's are indeterminates of degree 1 , and $I$ is a homogeneous ideal with this gradation. Since the line bundle for $E$ is given by $\left(f_{j} / x_{i}^{d}\right)^{-1} \mathscr{O}_{V_{i j}}$ on the affine open $V_{i j}$ where $\alpha_{j} \neq 0$, (4) is the height function if and only if

$$
\max \left(0, \quad\left[\max _{\left\{i^{\prime}: x_{i^{\prime}} \neq 0\right\}} \min _{j^{\prime}} v\left(f_{j^{\prime}} / x_{i^{\prime}}^{d}\right)\right]-v\left(f_{j} / x_{i}^{d}\right)\right)
$$

is bounded above and below on subsets of $V_{i j}$ where $\left|x_{i^{\prime}} / x_{i}\right|$ and $\left|\alpha_{j^{\prime}} / \alpha_{j}\right|$ are bounded by $e^{\gamma(v)}$ for an $M_{k}$-constant $\gamma(v)$. This is easy to check, using the relation $f_{j^{\prime}} \alpha_{j}=f_{j} \alpha_{j^{\prime}}$.

Example 6. When we blow up $\mathbb{P}^{n}$ over $k=\mathbb{Q}$ along the subvariety defined by homogenous polynomials $f_{1}, \ldots, f_{r} \in \mathbb{Z}\left[x_{0}, \ldots, x_{n}\right]$, (4) shows that $\lambda\left(x,|\cdot|_{p}\right)$ is the logarithm of the highest power of $p$ that divides all of $f_{1}\left(x_{0}, \ldots, x_{n}\right), \ldots, f_{t}\left(x_{0}, \ldots, x_{n}\right)$, where we choose $x_{i}$ to be in $\mathbb{Z}$ with no common divisors. Therefore, as in [14, the sum of all non-archimedean local heights is

$$
\log \operatorname{gcd}\left(f_{1}(x), \ldots, f_{r}(x)\right) \text {. }
$$

The archimedean local height is not a standard gcd: if $E$ is the exceptional divisor on the blowup of $\mathbb{P}^{2}$ at $[0: 0: 1]$, then

$$
\lambda_{E}\left([2: 3: N],|\cdot|_{\infty}\right)=\min \left(-\log \frac{2}{N},-\log \frac{3}{N}\right)=\log N-\log 3
$$


Example 7. In this paper, we deal with blowups of surfaces, so the blowups occur at points. By choosing an open cover so that only one open set $U$ contains the blowup point, (4) simply becomes

$$
\lambda(P, v)= \begin{cases}\max \left(0, \min _{j} v\left(f_{j}(P)\right)\right) & P \in U, \\ 0 & \text { otherwise, }\end{cases}
$$

where $f_{j}$ are the local equations of the blowup point in $U$.

If all we need is some bound for the height function on the blowups rather than exact expressions, then we do not need the local generators. One could work with Nakai-Moishezon criterion, but the following naïve estimate suffices.

Proposition 8. Let $X$ be a projective scheme over $k$, let $H$ be a very ample divisor, and let $\mathscr{I}$ define a closed subscheme. Let $\pi: \widetilde{X} \longrightarrow X$ be the blowup of $X$ along $\mathscr{I}$, and let $E$ be the exceptional divisor. Then there exists a very ample divisor $A$ of $\tilde{X}$ such that

for all $P \in \widetilde{X}(k)$.

$$
h_{H}(\pi(P)) \leq h_{A}(P)+O(1)
$$

Proof. Let $\mathscr{O}_{X}(1)$ be the line bundle corresponding to $H$. If $m+1$ global sections generate $\mathscr{I} \otimes \mathscr{O}_{X}(d)$, we have

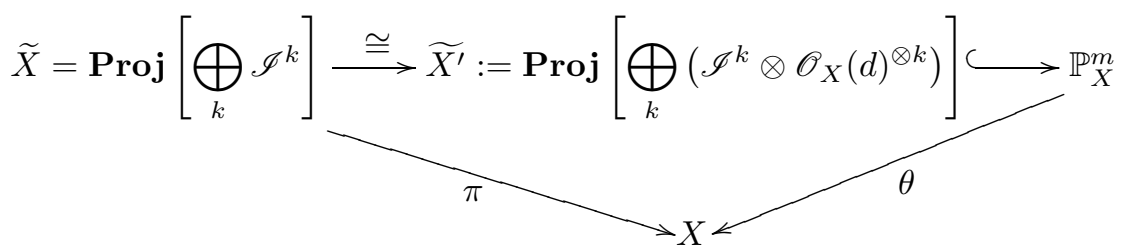

As in [7, Lemma II.7.10], $\mathscr{O}_{\mathbb{X}}(1)$ pulls back to $\mathscr{O}_{\widetilde{X}}(1) \otimes \pi^{*} \mathscr{O}_{X}(d)$ on $\tilde{X}$. Since $\theta^{*} \mathscr{O}_{X}(1) \otimes \mathscr{O}_{\mathbb{P}_{X}^{m}}(1)$ is very ample on $\mathbb{P}_{X}^{m}$ and $\mathscr{L}(E)=\mathscr{O}_{\widetilde{X}}(-1),-E+(d+1) H$ is very ample. So we can let $A$ to be $-E+(d+2) H$.

2.1. Local heights of multiple blowups of $\mathbb{P}^{2}$. We will specifically compute heights in important special cases, to be used in the proof of Theorem 1. We start the blowup at $[0: 0: 1]$ of $\mathbb{P}^{2}$. We then always blow up at the most recently created exceptional divisor, that is, $X^{(n)}$ is the blowup of $X^{(n-1)}$ at a point $P_{n-1}$ on $E^{(n-1)}$. The composition $\pi_{n 0}$ of blowups is an isomorphism over $\mathbb{P}^{2} \backslash[0: 0: 1]$. We will continue to use the notation used in the proof of Proposition 5 .

We let $[X: Y: Z]$ be the coordinates in $\mathbb{P}^{2}$, and let $x=X / Z$ and $y=Y / Z$. Over an open set containing $P_{0}=[0: 0: 1]$,

$$
\operatorname{Proj} k[x, y]\left[\alpha_{1}: \beta_{1}\right] /\left(x \beta_{1}-y \alpha_{1}\right)
$$

represents $X^{(1)}$. For notational convenience, let $\theta_{0}=x$ and $\varphi_{0}=y$.

To compute heights as we continue the blowup process, assume that $P_{i}$ has a coordinate $\left[a_{i}: b_{i}\right]$ on $E^{(i)}$. Let us define (inductively for $\varphi_{i}$ )

$$
\begin{aligned}
& \Phi_{i}: \text { a linear form in two variables which is nonzero at }\left(a_{i}, b_{i}\right), \\
& \theta_{i}=\frac{a_{i} \beta_{i}-b_{i} \alpha_{i}}{\Phi_{i}\left(\alpha_{i}, \beta_{i}\right)} \\
& \varphi_{i}=\Phi_{i}\left(\theta_{i-1}, \varphi_{i-1}\right) .
\end{aligned}
$$


Note that $\theta_{i}$ is a rational function of $\alpha_{i}$ and $\beta_{i}$ which is an affine coordinate on $E^{(i)}$ around $P_{i}$, and $\varphi_{i}$ is a linear form in $x, y, \theta_{1}, \ldots, \theta_{i-1}$. With this notation, it is easy to prove by induction that the ideal sheaf of the point $P_{n-1}$ inside $X^{(n-1)}$ is generated locally by $\theta_{n-1}$ and $\varphi_{n-1}$, and that on an open set containing $E^{(n)}$, $X^{(n)}$ is given by

$$
\operatorname{Proj} k\left[x, y, \theta_{1}, \ldots, \theta_{n-1}\right]\left[\alpha_{n}: \beta_{n}\right] /\left(\theta_{0} \beta_{1}-\varphi_{0} \alpha_{1}, \ldots, \theta_{n-1} \beta_{n}-\varphi_{n-1} \alpha_{n}\right) .
$$

Using the relations in (77) inductively, we can express $\alpha_{i}, \beta_{i}, \theta_{i}, \varphi_{i}$ as rational functions of $x$ and $y$ (with coefficients involving $a_{1}, \ldots, a_{n}$ and $b_{1}, \ldots, b_{n}$ ) for all $i$. From (17) and Example [7, we have

Proposition 9. With the notation of the previous paragraph, local height function on $X^{(n)}$ with respect to $E^{(n)}$ is

$$
\lambda_{E^{(n)}}(P, v)=\max \left(0, \min \left(v\left(\theta_{n-1}(P)\right), v\left(\varphi_{n-1}(P)\right)\right)\right)
$$

for $P$ outside of $E^{(n)}$.

Example 10. Suppose we always blow up at [0:1] or [1:0] on the exceptional divisor in the coordinates of (7). Without loss of generality let $X^{(2)}$ be the blowup of $X^{(1)}$ at $[0: 1]$. Then let $\Phi_{1}\left(\alpha_{1}, \beta_{1}\right)=\beta_{1}$, and $\theta_{1}=-\frac{\alpha_{1}}{\beta_{1}}=-\frac{x}{y}$. Then by (8),

$$
\lambda_{X^{(2)}, E^{(2)}}([x: y: 1], v)=\max (0, \min [v(x / y), v(y)]) .
$$

This is positive if and only if $0<v(y)<v(x)$, which can be conveniently written as $\frac{0}{1} v(x)<v(y)<\frac{1}{1} v(x)$. We will prove by induction that $\theta_{n-1}=\frac{x^{e}}{y^{f}}$ and $\varphi_{n-1}=\frac{y^{d}}{x^{c}}$ or the other way around for some nonnegative integers $c, d, e, f$. If we blow up at $[0: 1]$ on $E^{(n)}$, we can let $\Phi_{n}\left(\alpha_{n}, \beta_{n}\right)=\beta_{n}$. Using the relation $\theta_{n-1} \beta_{n}-\varphi_{n-1} \alpha_{n}$, we have

$$
\theta_{n}=-\frac{\alpha_{n}}{\beta_{n}}=-\frac{\theta_{n-1}}{\varphi_{n-1}}=-\frac{x^{c+e}}{y^{d+f}}, \quad \varphi_{n}=\varphi_{n-1}=\frac{y^{d}}{x^{c}} .
$$

Similarly, if we blow up at $[1: 0]$, then $\theta_{n}=\frac{y^{d+f}}{x^{c+e}}$ and $\varphi_{n}=\frac{x^{e}}{y^{f}}$. If $\theta_{n-1}$ and $\varphi_{n-1}$ are reversed, then the above formulae for $[0: 1]$ and $[1: 0]$ blowups get interchanged. Thus, induction goes through, and by Proposition 9, the local height on $X^{(n)}$ is

$$
\lambda_{X^{(n)}, E^{(n)}}([x: y: 1], v)=\max \left(0, \min \left[v\left(x^{e} / y^{f}\right), v\left(y^{d} / x^{c}\right)\right]\right)
$$

for $[x: y: 1] \in \mathbb{P}^{2} \backslash(X Y Z)$, so the height is positive only if $\frac{c}{d} v(x)<v(y)<\frac{e}{f} v(x)$.

The range of $v(y) / v(x)$ that gives a positive height changes from $\left(\frac{c}{d}, \frac{e}{f}\right)$ to $\left(\frac{c}{d}, \frac{c+e}{d+f}\right)$ or $\left(\frac{c+e}{d+f}, \frac{e}{f}\right)$ upon a blowup, so these are Farey sequences. In particular, $d e-c f=1$ throughout this process. The height formulae are organized in the tree on the next page (known as a Scott-Brocot tree). For each $\bullet$ representing the variety, the local height is positive only if $v(y) / v(x)$ is between the two fractions that bound the $\bullet$. Every time we blow up at [1:0], the role of $\theta$ and $\varphi$ flip, so from the root of the tree, if it takes an odd number of $[1: 0]$ blowups to reach a certain $\bullet$, then the next blowup at $[0: 1]$ goes to the right in the tree and the blowup at $[1: 0]$ goes to the left. If it takes an even number of $[1: 0]$ blowups to reach a certain -, then the next $[0: 1]$ blowup goes to the left and the next [1:0] blowup goes to the right. The points labeled $[0: 1]$ and $[1: 0]$ are special geometrically, because these are precisely the points where the strict transforms of previous exceptional divisors intersect the newest exceptional divisor. To see this, our formula always 


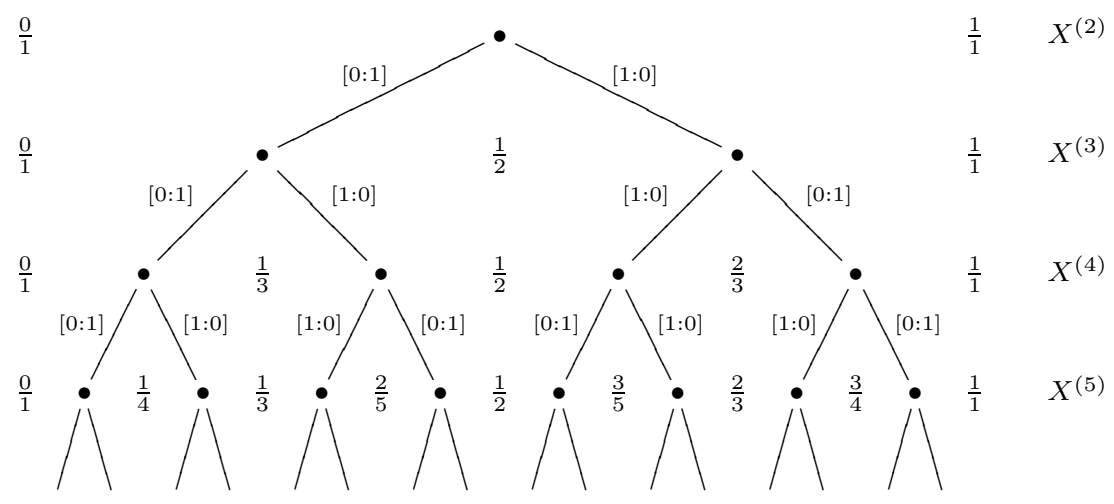

has the form $\operatorname{Proj} A[\alpha: \beta] /(f \beta-g \alpha)$, where $(f, g)$ is a regular sequence defining the smooth blowup point and $A$ is an affine open around it. The point $[0: 1]$ is cut out by $\frac{\alpha}{\beta}$ and $g$ locally, and the zero locus of $\frac{\alpha}{\beta}$ is the strict transform of $f=0$ while the zero locus of $g$ is the exceptional divisor. The point $[1: 0]$ is similar, and so $[0: 1]$ and $[1: 0]$ on the exceptional divisor are precisely the points where the exceptional divisor meets the strict transforms of the generators from the previous stage. By induction, on $X^{(n)}$, exactly two of $\left\{\widetilde{(X)}, \widetilde{(Y)}, E^{(1, n)}, \ldots, E^{((n-1), n)}\right\}$ meet $E^{(n)}$ and $[0: 1]$ and $[1: 0]$ are the intersection points. In fact, $X^{(n)}$ constructed this way is always a toric variety. We will see in Proposition 13 that the geometric property of toricness corresponds to the arithmetic property of having particularly simple local height expressions (9) which are "big" on $S$-units.

\section{Proof of Theorem 1}

We use the notation of Section 1.1 right before Theorem 1 . We can find an element $\varphi \in \mathrm{GL}_{3}(\bar{k})$ which sends $L$ to the three lines $X Y Z=0$, and by possibly enlarging $k$ and $S$, it suffices to prove the theorem for $S$-integral points of $\mathbb{P}^{2} \backslash(X Y Z)$. Thus, we can let $L=X Y Z$ and

$$
I=\left\{[x: y: 1] \in \mathbb{P}^{2}-|L|: x, y \in \mathcal{O}_{S}^{*}\right\}=\left\{[X: Y: Z] \in \mathbb{P}^{2}-|L|: X, Y, Z \in \mathcal{O}_{S}^{*}\right\} .
$$

For convenience, $h$ without any specified divisor will mean the usual Weil height function on projective spaces with respect to a hyperplane.

To prove the theorem, for each $m \leq n$ we will analyze how $E^{(m)} \subseteq X^{(m)}$ is constructed geometrically and determine whether $h_{E^{(m, n)}}$ is "small" (bounded above by $\epsilon h_{A}$ ) or not on $I$. We will then show that "big" ones occur with a negative coefficient in the canonical divisor.

Let us fix $m \leq n$ and describe the "relevant" subtower of blowups for creating $E^{(m)}$ in $X^{(m)}$. We can show by induction that set-theoretically

$$
X^{(m-1)}=\left(\mathbb{P}^{2}-B\right) \cup E^{(1,(m-1))} \cup \cdots \cup E^{(m-1)} .
$$

There is no triple intersection among the components in (10), and the intersection multiplicity between any two components is one. Therefore, the point $\pi_{m, m-1}\left(E^{(m)}\right)$ $=P_{m-1}$ lies in at least one and at most two of the $E^{(j, m-1)}$ for $j=0, \ldots, m-1$, where we let $E^{(0, m-1)}=\mathbb{P}^{2}-B$ for notational convenience. Define the predecessor of $m$ to be the largest $j$ such that $E^{(j, m-1)}$ contains $\pi_{m, m-1}\left(E^{(m)}\right)$. Next we take 
$X^{(5)}$
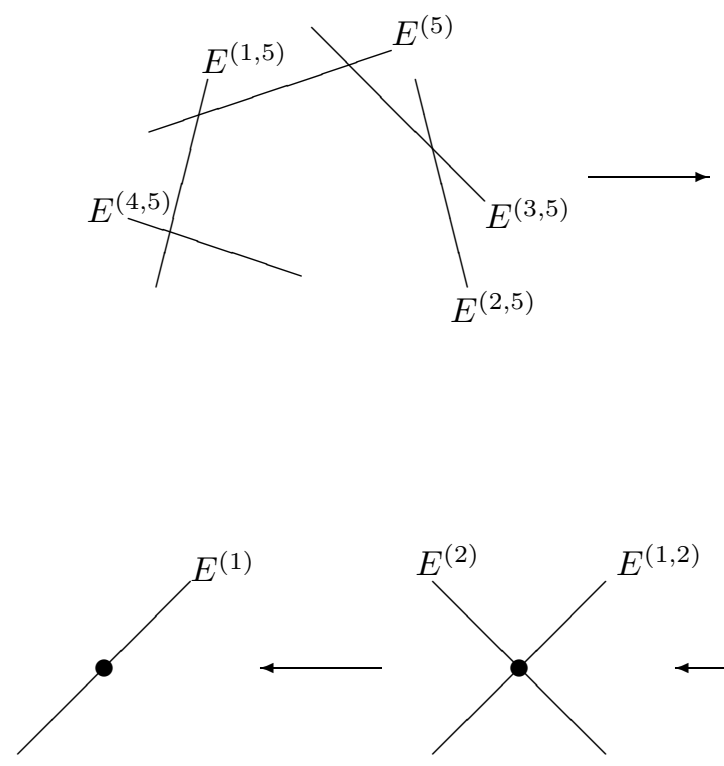

$X^{(1)}$

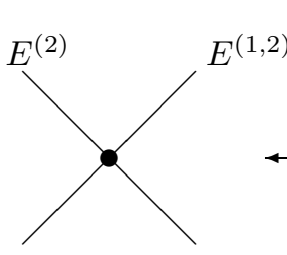

$X^{(2)}$
$X^{(4)}$
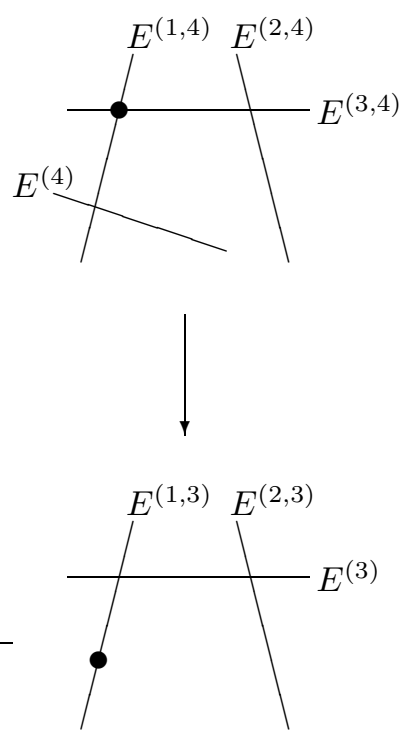

$X^{(3)}$

the predecessor of the predecessor, continuing the process until we reach the index 0 . The ordered set of indices that we hereby obtain from 0 to $m$ is the relevant subtower for $E^{(m)}$.

In the example drawn above, the circled point is the place we blow up to get the next one. For $E^{(5)}, P_{4} \in X^{(4)}$ lies in $E^{(1,4)}$ and $E^{(3,4)}$, so the predecessor is 3. Then $P_{2} \in X^{(2)}$ lies on $E^{(1,2)}$ and $E^{(2)}$, so the predecessor of 3 is 2 . So the relevant subtower for 5 is $0<1<2<3<5$. Since $E^{(3,5)}$ is not a result of blowing up directly from $X^{(1)}$ like $E^{(4,5)}$, we need 2 in the relevant subtower. The relevant subtower for 4 is just $0<1<4$.

The following proposition justifies the name "relevant subtower": we can actually do these blowups first and then fill in the skipped processes, preserving the geometric properties of $E^{(m, n)}$.

Proposition 11. (i) Let $j$ be the predecessor of $m$. For any $i$ with $j \leq i<m-1$, the point $\pi_{m i}\left(E^{(m)}\right) \in X^{(i)}$ is different from $P_{i}$.

(ii) Suppose that the relevant subtower for $m$ is $0=m_{0}<m_{1}<\cdots<m_{l}=m$. Then we can inductively define another sequence of blowups denoted $Y^{(i)}$ with corresponding exceptional divisor $F^{(i)}$ for $i=1, \ldots, l$ satisfying the following properties:

(1) $Y^{(i)}$ is the blowup of $Y^{(i-1)}$ at a point on $F^{(i-1)}$ (i.e., on the most recently constructed exceptional divisor).

(2) The pullback of $F^{(i)}$ to $Y^{(l)}$ is a strictly positive linear combination of $F^{(i, l)}, \ldots, F^{(l)}$.

(3) There exists a sequence of $\left(m_{i}-i+1\right)$ blowups $\theta_{i}: X^{\left(m_{i}\right)} \longrightarrow Y^{(i)}$ such that $\theta_{i}$ is an isomorphism on an open set containing $E^{\left(m_{i}\right)}$, identity on $\mathbb{P}^{2}-B$, and $\theta_{i}^{*}\left(F^{(i)}\right)=E^{\left(m_{i}\right)}$. 
Proof. (i) By definition, $\pi_{m, m-1}\left(E^{(m)}\right)$ is not in $E^{(i+1, m-1)}, \ldots, E^{(m-1)}$. On the other hand, the inverse image of $E^{(i+1)}$ to $X^{(m-1)}$ is contained in the union of $E^{(i+1, m-1)}, \ldots, E^{(m-1)}$. Therefore, $\pi_{m, i+1}\left(E^{(m)}\right)$ is not in $E^{(i+1)}$, hence $\pi_{m i}\left(E^{(m)}\right)$ is different from $P_{i}$.

(ii) The base case of induction is $i=0$ and $\theta_{0}=\mathrm{id}$. Suppose we have already constructed $\theta_{i}: X^{\left(m_{i}\right)} \longrightarrow Y^{(i)}$. The image of $E^{\left(m_{i}, m_{i+1}-1\right)}$ via $\pi_{m_{i+1}-1, m_{i}}$ is $E^{\left(m_{i}\right)}$, so $P=\pi_{m_{i+1}, m_{i}}\left(E^{\left(m_{i+1}\right)}\right)$ is in $E^{\left(m_{i}\right)}$. By induction, $\theta_{i}$ is an isomorphism on an open set containing $E^{\left(m_{i}\right)}$. Since the construction of blowups is local, we get the map on the top row in the following diagram

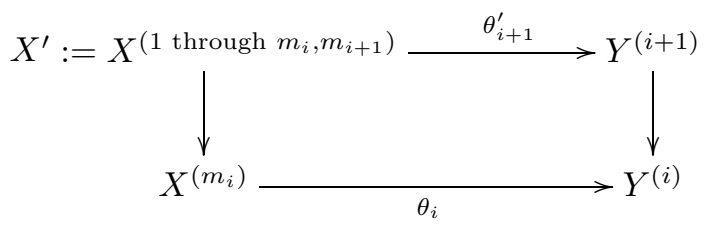

where the left vertical map is the blowup at $P$ and the right vertical map is the blowup at $\theta_{i}(P)$. Note that $\theta_{i+1}^{\prime}$ is an isomorphism on an open set containing the exceptional divisor from the latest blowup, which is $\left(\theta_{i+1}^{\prime}\right)^{*}\left(F^{(i+1)}\right)$. We now use (i) repeatedly. Since $P_{m_{i}}$ and $P$ are two different points of $X^{\left(m_{i}\right)}$, the order of these two blowups can be interchanged. We can then exchange the order of two blowups on $X^{\left(m_{i}+1\right)}$, as $P_{m_{i}+1} \neq \pi_{m_{i+1}, m_{i}+1}\left(E^{\left(m_{i+1}\right)}\right)$ by (i). In the end, we will have $X^{\left(m_{i+1}\right)} \rightarrow X^{\prime}$. Composing with $\theta_{i+1}^{\prime}$, the pullback of $F^{(i+1)}$ is exactly $E^{\left(m_{i+1}\right)}$, and this is the desired $\theta_{i+1}$. Properties (1) and (2) are clear by construction.

The following allows us to reduce height consideration to the relevant subtower:

Corollary 12. Let $m \leq n, m_{i}$ be an index in the relevant subtower for $m$. Let $Y^{(i)}$ and $F^{(i)}$ be as in Proposition 11, and let $\varpi_{i}: Y^{(i)} \longrightarrow \mathbb{P}^{2}$ be the composition of the blowups. Suppose that given $\epsilon>0$, there exists a Zariski-closed $Z_{\epsilon} \subsetneq \mathbb{P}^{2}$ containing $B$ such that

$$
h_{F^{(i)}, Y^{(i)}}\left(\varpi_{i}^{-1}(P)\right)<\epsilon h(P)+O(1)
$$

for $P \in I \backslash Z_{\epsilon}$. Then given $\epsilon>0$, for all $P \in I \backslash Z_{\epsilon}$,

$$
h_{E^{(m, n)}, X^{(n)}}\left(\pi_{n 0}^{-1}(P)\right)<\epsilon h(P)+O(1) .
$$

Proof. Let $m=m_{l}$. By property (2) in Proposition 11] and by functoriality of heights, we can replace the left-hand side of (11) by a strictly positive linear combination of heights with respect to $F^{(i, l)}, F^{((i+1), l)}, \ldots, F^{(l)}$. Since the height with respect to an effective divisor is nonnegative away from the divisor, this implies

$$
h_{F^{(l)}, Y^{(l)}}\left(\varpi_{l}^{-1}(P)\right)<\epsilon h(P)+O(1)
$$

for $P \in I \backslash Z_{\epsilon}$. By property (3), this in turn implies

$$
h_{E^{(m)}, X^{(m)}}\left(\pi_{m 0}^{-1}(P)\right)<\epsilon h(P)+O(1)
$$

for $P \in I \backslash Z_{\epsilon}$. Moreover, $\pi_{n m}^{*}\left(E^{(m)}\right)$ is $E^{(m, n)}$ plus possibly other effective divisors, so we are done by the same argument.

Therefore, for each $m \leq n$, if there is one index in the sequence of predecessors where the height is small on $I, h_{E^{(m, n)}}$ is also small. Next, we determine when there is such an index. When the relevant subtower of $m$ is $0<m_{1}<\cdots<m_{l}=m$, for 
each $Y^{(i)}$, consider the set $\mathfrak{S}_{i}$ which consists of the strict transforms of the three lines and exceptional divisors $F^{(1, i)}, \ldots, F^{(i)}$. If the point we blow up to construct $Y^{(i+1)}$ is at the intersection of two divisors in $\mathfrak{S}_{i}$ for all $i=0, \ldots, l-1$, then we say $E^{(m, n)}$ is type two; else $E^{(m, n)}$ is type one. In other words, in the relevant subtower, if the blowup always occurs at an intersection of lines and previously constructed exceptional divisors, $E^{(m, n)}$ is type two. In particular, if $E^{(m, n)}$ is type two, the first blowup in the relevant subtower must be at one of the three points of intersection of the triangle. Moreover, note that even if the blowup point for constructing $Y^{(l)}$ occurs at an intersection point, if one of the previous blowups in the relevant subtower occurs off of the intersection points, then $E^{(m, n)}$ is type one. The following proposition demonstrates some properties of type two divisors, including the fact that heights with respect to these are big on $I$. This is in a sharp contrast with type one divisors, as we will see in Proposition 15.

Proposition 13. Suppose that $E^{(m, n)}$ is type two.

(i) There exists a strictly positive constant $C$ such that

$$
h_{E^{(m)}, X^{(m)}}\left(\pi_{m 0}^{-1}(P)\right) \geq C \cdot h(P)+O(1)
$$

for a Zariski-dense set of points $P$ of $I$.

(ii) We can write $K^{(n)}=-\widetilde{L}-E^{(m, n)}+$ other components.

Remark. (i) shows that type two divisors cannot satisfy the hypothesis of Corollary 12 so the height is big on $I$. The relevant subtower is a toric variety in this case, since the blowup always occurs at a fixed point of the torus action. So (ii) also follows from the theory of toric varieties, as the canonical divisor is the negative of the sum of the $T$-divisors [6]. We will give a more direct proof here.

Proof. (i) By property (3) of Proposition [11, the left-hand side can be replaced by $h_{F^{(l)}, Y^{(l)}}$. By assumption, every blowup in the process of constructing $Y^{(l)}$ occurs at a point of intersection. So without loss of generality, we can assume that $Y^{(1)}$ is the blowup at $[0: 0: 1]$, and from Example 10 .

$$
h_{F^{(l)}, Y^{(l)}}([x: y: 1])=\sum_{v} \max \left(0, \min \left[v\left(x^{e} / y^{f}\right), v\left(y^{d} / x^{c}\right)\right]\right),
$$

where $\frac{c}{d}<\frac{e}{f}$ is a Farey pair of order $l$ (starting with $\frac{0}{1}<\frac{1}{0}$ for $Y^{(1)}$ ). Given $\delta>0$, choose $\frac{\mu}{\rho}$ to be any reduced fraction between $\frac{c+e}{d+f}-\delta$ and $\frac{c+e}{d+f}$. The rational curve $x^{\mu}=y^{\rho}$ has infinitely many points of $I$, and (12) is at least

$$
\left(d \cdot \frac{\mu}{\rho}-c\right) v(x)>\left(d\left(\frac{c+e}{d+f}-\delta\right)-c\right) v(x)=\left(\frac{1}{d+f}-d \delta\right) v(x)
$$

when $v(x)>0$ (the first term of (12) is larger). On this curve, $|v(x)| \geq|v(y)|$, so

$$
h([x: y: 1])=h([1: y / x: 1 / x])=\sum_{v, v(x)>0} \max (0, v(x)-v(y), v(x))=\sum_{v, v(x)>0} v(x) .
$$

Therefore, choosing an appropriate $\delta$,

$$
h_{F^{(l)}, Y^{(l)}}([x: y: 1]) \geq \frac{1}{d+f+1} h([x: y: 1])
$$

on infinitely many curves, each of which contains infinitely many points of $I$.

(ii) Since each blowup point is on two components, by exercise II.8.5 of [7], it is easy to show by induction that the canonical divisor for $Y^{(l)}$ can be written 
as $-\widetilde{L}-F^{(1, l)}-\cdots-F^{(l)}$. By property (3) of Proposition [1], $K^{(m)}$ is then $-\widetilde{L}-E^{(m)}$ plus other components. By applying the same exercise to each blowup of $X^{(n)} \longrightarrow X^{(m)}$, we are done.

Next we show that the height with respect to a type one exceptional divisor is small on $I$. This is where we use the following result of Corvaja and Zannier [3].

Theorem 14. Let $\Gamma \subset \mathbb{G}_{m}^{2}(\overline{\mathbb{Q}})$ be a finitely generated group, let $p, q$ be nonconstant coprime polynomials in two variables with coefficients in $\overline{\mathbb{Q}}$, and suppose that not both of them vanish at $(0,0)$. For every positive $\epsilon$,

$$
\sum_{v \in M_{k}} \max (0, \min [v(p(t, u)), v(q(t, u))])<\epsilon \max (h(t), h(u))
$$

holds for $(t, u) \in \Gamma$ outside a Zariski-closed $Z_{\epsilon} \subsetneq \mathbb{G}_{m}^{2}$.

Silverman 14 noted that this was the essential information for showing Vojta's conjecture on one blowup of $\mathbb{P}^{2}$ for a triangular divisor, restricted to integral points. We will generalize this observation to multiple blowups in the following proposition, using Example 10. Combined with Corollary 12, the next proposition implies that the height with respect to a type one exceptional divisor is small on $I$.

Proposition 15. Suppose $E^{(m, n)}$ is such that in its relevant subtower $0<m_{1}<$ $\cdots<m_{l}$, all $F^{(i)}$ for $i<l$ are type two but $F^{(l)}$ is type one. Then given $\epsilon>0$, there exists a Zariski-closed $Z_{\epsilon} \subsetneq \mathbb{P}^{2}$ containing $B$ such that

$$
h_{F^{(l)}, Y^{(l)}}\left(\varpi_{l}^{-1}(P)\right)<\epsilon h(P)+O(1)
$$

for $P \in I \backslash Z_{\epsilon}$. And the same inequality holds for the height of $E^{(m, n)}$.

Remark. From the proof of Theorem 1 given below, it will be clear that this proposition is necessary for Theorem 1 to hold (see also Example 2).

Proof. Without loss of generality, we may assume that $Y^{(1)}$ is the blowup of $\mathbb{P}^{2}$ at a point not on $Z=0$, say $[a: b: 1]$. If $m=m_{1}$, then by definition of type one, $(a, b) \neq(0,0)$. By (8) $)$, we have

$$
h_{F^{(1)}, Y^{(1)}}([x: y: 1])=\sum_{v \in M_{k}} \max (0, \min (v(x-a), v(y-b))) .
$$

By Theorem 14, given $\epsilon>0$, there exists a proper Zariski-closed $Z_{\epsilon}$ such that

$$
h_{F^{(1)}, Y^{(1)}}(P)<\epsilon \max (h(x), h(y))
$$

holds for $P=[x: y: 1] \in I \backslash Z_{\epsilon}$. So we are done in this case because

$$
\max (h(x), h(y))=\max \left(\sum_{v} \max (v(x), 0), \sum_{v} \max (v(y), 0)\right) \leq h([x: y: 1]) .
$$

If $m \neq m_{1}$, then we have some type two exceptional divisors in the relevant subtower for $m$. Using the notation from Example 10 for $Y^{(l-1)}$, we have $\theta_{l-2}=\frac{x^{e}}{y^{f}}$ and $\varphi_{l-2}=\frac{y^{d}}{x^{c}}$ or vice versa. Let us assume the former without loss of generality. By assumption, $Y^{(l)}$ is the blowup of $Y^{(l-1)}$ at a point on $F^{(l-1)}$ not $[1: 0]$ or $[0: 1]$, so say $\left[a^{\prime}: 1\right]$ with $a^{\prime} \neq 0$. In the notation of Section 2.1, choosing the linear form $\Phi_{l-1}$ to be the second variable, we have

$$
\theta_{l-1}=\frac{a^{\prime} \varphi_{l-2}-\theta_{l-2}}{\varphi_{l-2}}=a^{\prime}-\frac{\theta_{l-2}}{\varphi_{l-2}}, \quad \varphi_{l-1}=\varphi_{l-2} .
$$


If $x, y \in \mathcal{O}_{S}^{*}$, then so are $\theta_{l-2}$ and $\varphi_{l-2}$, so the height computed in Proposition 9

$$
h_{F^{(l)}, Y^{(l)}}=\sum_{v} \max \left(0, \min \left[v\left(a^{\prime}-\frac{\theta_{l-2}}{\varphi_{l-2}}\right), v\left(\varphi_{l-2}\right)\right]\right)
$$

can be thought of as a gcd of polynomials in $\frac{\theta_{l-2}}{\varphi_{l-2}}, \varphi_{l-2} \in \mathcal{O}_{S}^{*}$. Hence, by Theorem 14 this is bounded above by

$$
\epsilon \max \left(h\left(\theta_{l-2} / \varphi_{l-2}\right), h\left(\varphi_{l-2}\right)\right)+O(1),
$$

except for points satisfying a polynomial in terms of these two variables. We can rewrite the exceptional set as a polynomial in $x$ and $y$, so this upper bound is valid for all points of $I$ outside of a proper Zariski-closed set in $\mathbb{P}^{2}$. Moreover,

$$
\max \left(h\left(\theta_{l-2} / \varphi_{l-2}\right), h\left(\varphi_{l-2}\right)\right) \leq h\left(\theta_{l-2}\right)+h\left(\varphi_{l-2}\right) \leq 2 \max (c, d, e, f) h([x: y: 1]) .
$$

So we are done by adjusting $\epsilon$.

Now we can finish the proof of Theorem 1, It always suffices to check Vojta's conjecture for one ample divisor. By induction and repeatedly applying Proposition 8, there exists a very ample divisor $A$ on $X^{(n)}$ such that $h_{A}(P)$ is at least $h(P)$ (up to a bounded function). Therefore, for any $\epsilon>0$, if we can bound the left-hand side of the inequality (1) in the theorem by $\epsilon h(P)$, we are done.

Writing $K^{(n)}$ as $-\widetilde{L}$ plus linear combinations of $E^{(1, n)}, \ldots, E^{(n)}$, let $N$ be the largest coefficient that appears (we do not need a bound for $N$, but $N$ is at most $(n+1)$-st Fibonacci number). We look at the left-hand side of the inequality component-wise. First,

$$
\sum_{v \in S} \lambda_{\widetilde{L}}(P, v)+h_{-\widetilde{L}}(P)=-\sum_{v \notin S} \lambda_{\widetilde{L}}(P, v),
$$

so this is nonnegative (up to a bounded function). For $m \leq n$ such that $E^{(m, n)}$ is type two, then by Proposition 13 (ii), the coefficient of $E^{(m, n)}$ in the expression of $K^{(n)}$ is -1 , and since $D$ contains at most one copy of $E^{(m, n)}$ by the normal crossings condition, the height coming from this component will be bounded above by a bounded function. For $m \leq n$ such that $E^{(m, n)}$ is type one, construct the relevant subtower $0<m_{1}<\cdots<m_{l}=m$, and let $m_{i}$ be the smallest index that is type one $(1 \leq i \leq l)$. Applying Proposition 15 with $\frac{\epsilon}{(N+1) n}$, there exists a Zariski-closed $Z_{\epsilon, m} \subsetneq \mathbb{P}^{2}$ such that

$$
h_{F^{(i)}, Y^{(i)}}(P)<\frac{\epsilon}{(N+1) n} h(P)+O(1)
$$

for $P \in I \backslash Z_{\epsilon, m}$. By Corollary 12, we can replace the left-hand side of (14) by $h_{E^{(m, n)}, X^{(n)}}\left(\pi_{n 0}^{-1}(P)\right)$. We also have

$$
\sum_{v \in S} \lambda_{E^{(m, n)}}(P, v) \leq h_{E^{(m, n)}}(P)+O(1)
$$

so the total contribution from the height of $E^{(m, n)}$ is at most $\frac{\epsilon}{n} h(P)$. Combining different components, we now see that the left-hand side of (11) is bounded above by $\epsilon h(P)$, as long as $P \in I$ avoiding all of the $Z_{\epsilon, m}$ and $B$. This concludes the proof. 


\section{Extensions}

In this section, we discuss extensions of Theorem 1, both provable and open. First, we generalize the inductive step of Theorem 1 . The key in the inductive argument was finding a divisor relative to which height of integral points is small, and then using functoriality of heights to show that the smallness is kept on subsequent blowups. We can generalize this idea to show that in some cases Vojta's conjecture on the base variety implies Vojta's conjecture for the blowup. Of course, we still have to know Vojta's conjecture on the base, but this shows that sometimes a seemingly stronger conjecture on the blowup is implied by the one on the original variety.

Proposition 16. Let $X$ be a smooth projective variety over $k$, let $Y$ be a smooth subvariety of codimension at least 2 , and let $\pi: \tilde{X} \longrightarrow X$ be the blowup of $X$ along $Y$. Suppose that we can write the canonical divisor $K$ of $X$ as a difference of nonnegative divisors $K=K_{+}-K_{-}$. Let $I$ be a set of $S$-integral points with respect to $K_{-}$. Let $D$ be a nonzero normal crossings divisor of the form $K_{-}+F$, where $F$ is a nonnegative divisor. Let $\left\{F_{i}\right\}$ be the irreducible components of $K_{+}+F$, and assume that $Y \subseteq\left|K_{+}+F\right|$. Further, if $F_{i} \nsubseteq\left|K_{+}\right|$, assume that

$$
\sum_{v \notin S} \lambda_{F_{i}}(P, v) \leq O(1)
$$

for $P \in I$. Let $D^{\prime}$ be a normal crossings divisor on $\widetilde{X}$ of the form

$$
D^{\prime}=\widetilde{K_{-}}+\chi E+\sum \chi_{i} \widetilde{F}_{i}
$$

where $\chi$ and $\chi_{i}$ are either 0 or 1 . Then Vojta's conjecture on $X$ for $D$ (if not for all points, at least for points in I) implies Vojta's conjecture on $\widetilde{X}$ for $D^{\prime}$ for points in $\pi^{-1}(I \backslash|Y|)$.

Remark. As stated in the introduction, Vojta's conjecture on a single blowup of $\mathbb{P}^{2}$ for a triangular divisor (Corvaja-Zannier case) is not a direct consequence of Vojta's conjecture for $\mathbb{P}^{2}$, and consistently, some hypotheses of this proposition are not satisfied. On the other hand, the proposition does apply to going from the first blowup to the second in the setting of Theorem 11, at least when $h_{E^{(1)}}$ is small (i.e., when $E^{(1)}$ is type one). So the proposition distinguishes the two kinds of blowups.

More specifically, when $X=\mathbb{P}^{2}, K_{-}=(X Y Z)$ and $K_{+}=0$, and if $F=0$, then $\left|K_{+}+F\right|$ is empty. If $F$ is a positive divisor on $\mathbb{P}^{2}$, then the normal crossings condition for $K_{-}+F$ implies that $F$ cannot go through $(0,0)$, so the dehomogenized polynomial defining $F$ has a nonzero constant. Then one can show that (15) contradicts the $S$-unit equation. So there is no way to satisfy the assumptions of the proposition.

On the other hand, if $X$ is the blowup of $\mathbb{P}^{2}$ off of the triangle and $Y$ is a point on $E^{(1)}$, the proposition applies with $F=0$. When $X^{(1)}$ is the blowup at a point on exactly one line, $F$ must be set to $E^{(1)}$. In this case, $\lambda_{E^{(1)}}([x: y: 1])=$ $\max (0, \min [v(x-1), v(y)])$, so (15) is satisfied, and the proposition applies. Thus, this proposition generalizes the inductive argument from $X^{(i)}$ when $E^{(i)}$ is a type one divisor (i.e., when $h_{E^{(i)}}$ is small). 
Proof. Fix an ample divisor $A$ on $X$. The left-hand side of Vojta's inequality for $X$ is

$$
\begin{aligned}
\sum_{v \in S} \lambda_{D}(P, v)+h_{K}(P) & =\sum_{v \in S}\left(\lambda_{K_{-}}(P, v)+\lambda_{F}(P, v)\right)-h_{K_{-}}(P)+h_{K_{+}}(P) \\
& =-\sum_{v \notin S} \lambda_{K_{-}}(P, v)+\sum_{v \in S} \lambda_{F}(P, v)+h_{K_{+}}(P)+O(1) .
\end{aligned}
$$

For points of $I$, the first term is a bounded function, so if we know Vojta's conjecture on $X$ for $D$ (for rational points or just for $I$ ), we conclude that

$$
\sum_{v \in S} \lambda_{F}(P, v)<\epsilon h_{A}(P)+O(1), \quad h_{K_{+}}(P)<\epsilon h_{A}(P)+O(1)
$$

for $P \in I$ except for a proper Zariski-closed subset $Z_{\epsilon}$. Therefore, using (15) when necessary, $h_{F_{i}}(P)<\epsilon h_{A}(P)+O(1)$ for each $i$. Since $\pi^{*}\left(F_{i}\right)$ is $\widetilde{F}_{i}$ plus possibly the exceptional divisor $E$, by functoriality, we have

$$
h_{\widetilde{F_{i}}}\left(\pi^{-1}(P)\right)<\epsilon h_{A}(P)+O(1)
$$

for $P \in I-Z_{\epsilon} \cup Y$. Moreover, since $\left|K_{+}+F\right|$ contains $Y, \pi^{*}\left(F_{i}\right)$ contains $E$ for some $i$, so the above inequality also holds when we replace the left-hand side by $h_{E}$. The canonical divisor of $\tilde{X}$ is linearly equivalent to $\pi^{*}(K)+(r-1) E$, where $r$ is the codimension of $Y$, so in our case, $K_{\widetilde{X}}=\widetilde{K_{+}}-\widetilde{K_{-}}+a E$ for some integer $a$. Therefore, the left-hand side of Vojta's inequality on $\widetilde{X}$ for $D^{\prime}$ becomes

$$
\begin{aligned}
& \sum_{v \in S} \lambda_{D^{\prime}}(Q, v)+h_{K_{\widetilde{X}}}(Q) \\
&= \sum_{v \in S}\left(\lambda_{\widetilde{K_{-}}}(Q, v)+\chi \lambda_{E}(Q, v)+\sum_{i} \chi_{i} \lambda_{\widetilde{F_{i}}}(Q, v)\right) \\
&-h_{\widetilde{K_{-}}}(Q)+h_{\widetilde{K_{+}}}(Q)+a h_{E}(Q) \\
&=-\sum_{v \notin S} \lambda_{\widetilde{K_{-}}}(Q, v)+\sum_{v \in S}\left(\begin{array}{c}
\left.\chi \lambda_{E}(Q, v)+\sum_{i} \chi_{i} \lambda_{\widetilde{F_{i}}}(Q, v)\right) \\
\quad+h_{\widetilde{K_{+}}}(Q)+a h_{E}(Q)
\end{array}\right. \\
& \leq-\sum_{v \notin S} \lambda_{\widetilde{K_{-}}}(Q, v)+(\chi+a) h_{E}(Q)+\sum_{i} a_{i} h_{\widetilde{F_{i}}}(Q) \\
& \leq(\chi+a) h_{E}(Q)+\sum_{i} a_{i} h_{\widetilde{F_{i}}}(Q)
\end{aligned}
$$

for some $a_{i}$ (the number of $F_{i}$ in $K_{+}$plus $\left.\chi_{i}\right)$. For $Q \in \pi^{-1}\left(I-Z_{\epsilon} \cup Y\right)$, both of these terms are bounded by a fixed multiple of $\epsilon h_{A}(\pi(Q))$ (plus a bounded function). As before, Proposition 8 finishes the proof.

We end the paper by discussing what happens to Theorem 1 when we change the divisor from a triangle or when we remove the integrality condition. This enables us to obtain new arithmetic results, unlike the case of Theorem 1, whose proof is essentially geometric and combinatorial once given the arithmetic input of [3]. So instead of a triangle, let $D$ be an irreducible cubic in $\mathbb{P}^{2}$ or the union of a line and an irreducible conic. If we blow up $\mathbb{P}^{2}$ at a point outside $|D|$, then Vojta's conjecture for the divisor $\widetilde{D}+E^{(1)}$ is still inequality (2) in Example 2, but we 
evaluate at integral points with respect to $D$. For example, if the divisor $D$ is the normal crossings divisor $Z\left(X Y-Z^{2}\right)=0$,

$$
I_{1}=\left\{(x, y): x, y \in \mathcal{O}_{S}, x y-1 \in \mathcal{O}_{S}^{*}\right\}
$$

is a set of $S$-integral points. Picking a point not on $|D|$, say $[1: 2: 1]$, we need to show

$$
\log \operatorname{gcd}^{+}(x-1, y-2)<\epsilon \max (h(x), h(y))+O(1)
$$

for $(x, y) \in I_{1}$ outside a finite union of curves, where

$$
\operatorname{gcd}^{+}(a, b)=\exp \left(\sum_{v \in M_{k}} \max (0, \min [v(a), v(b)])\right) .
$$

If the divisor is the elliptic curve $Y^{2} Z=X(X-Z)(X+Z)$, the set $I_{2}$ consisting of $[X: Y: Z] \in \mathbb{P}^{2}(k)$ satisfying

$$
v\left(Y^{2} Z-X(X-Z)(X+Z)\right)=3 \min (v(X), v(Y), v(Z)) \text { for } v \notin S
$$

is a set of $S$-integral points, and this can also be described as follows. Enlarging $S$ if necessary to make $\mathcal{O}_{S}$ into a principal ideal domain (PID), given a point $[X: Y: Z] \in I_{2}$, there exists a representative such that $X, Y, Z \in \mathcal{O}_{S}$ and $Y^{2} Z-X(X-Z)(X+Z) \in \mathcal{O}_{S}^{*}$. By extending $k$ and $S$ to include a cube root of each torsion of $\mathcal{O}_{S}^{*}$ and of each fundamental unit, we get a representative in $\mathcal{O}_{S}^{3}$ which is on the affine cubic hypersurface defined by $Y^{2} Z-X(X-Z)(X+Z)=1$. So Vojta's conjecture for integral points on the blowup of $\mathbb{P}^{2}$ at $[1: 2: 1]$ for this elliptic curve is

$$
\log \operatorname{gcd}^{+}\left(\frac{x}{z}-1, \frac{y}{z}-2\right)<\epsilon \max (h(x), h(y), h(z))+O(1)
$$

for $\left\{x, y, z \in \mathcal{O}_{S}: y^{2} z-x(x-z)(x+z)=1\right\}$ outside a finite union of curves. Both $I_{1}$ and $I_{2}$ are Zariski-dense sets which are not related to integral points of $\mathbb{G}_{m}^{2}$, so the inequalities (16) and (17) are fundamentally different from the situation of [3] and Theorem 1 ,

If we assume that the first blowup case holds for integral points with respect to such a $D$, then Proposition 16 does apply to show that the height with respect to any exceptional divisors from subsequent infinitely near blowups is small. So this part of the induction works. On the other hand, one of the keys in proving Theorem[1is Proposition 15] dealing with the first time the blowup occurs off of the intersections. In the case of the triangle, this is a variation of [3], as the condition of integrality with respect to a triangle ( $S$-unit condition) is preserved when we change variables $(x, y) \mapsto\left(x^{e} / y^{f}, y^{d} / x^{c}\right)$. A similar property is not immediately clear for integrality with respect to other cubic divisors, so even if we assume Vojta's conjecture for the first blowup for integral points, the multiple blowup case does not seem to follow.

We can also try removing or weakening the integrality condition in Theorem 1 . For example, we can look at the set of points which are integral with respect to two of the lines rather than integral to all three. If the blowup occurs off of the triangle 
$X Y Z=0$, say at $[1: 1: 1]$, the conjecture says

$$
\log \operatorname{gcd}^{+}(x-1, y-1)<\epsilon \max (h(x), h(y))+\sum_{v \notin S} v(y)+O(1)
$$

for $x \in \mathcal{O}_{S}^{*}$ and $y \in \mathcal{O}_{S}$. This seems to be difficult. On the other hand, if we blow up at a point on one line of the triangle, Vojta's conjecture in full (for all rational points) can be proved from Schmidt's subspace theorem. This result, together with its higher-dimensional and multiple-blowup generalization, is discussed in [16].

\section{ACKNOWLEDGMENTS}

This paper originated from a portion of the author's Ph.D. thesis [17 at Brown University, and he would like to thank his advisor, Joseph Silverman, for all of his help. He would also like to thank Dan Abramovich and Lucien Szpiro for many enlightening discussions, and the anonymous referee for numerous helpful comments. In addition, thanks go to the Fields Institute for their hospitality during a workshop on Arithmetic Geometry: Diophantine approximations and Arakelov theory.

\section{REFERENCES}

1. Enrico Bombieri and Walter Gubler, Heights in Diophantine geometry, New Mathematical Monographs, vol. 4, Cambridge University Press, Cambridge, 2006. MR2216774(2007a:11092)

2. Pietro Corvaja and Umberto Zannier, On integral points on surfaces, Ann. of Math. (2) 160 (2004), no. 2, 705-726. MR2123936 (2005m:11118)

3. $\quad$ A lower bound for the height of a rational function at $S$-unit points, Monatsh. Math. 144 (2005), no. 3, 203-224. MR2130274 (2005k:11140)

4. Gerd Faltings, Endlichkeitssätze für abelsche Varietäten über Zahlkörpern, Invent. Math. 73 (1983), no. 3, 349-366. MR718935 (85g:11026a)

5. , Diophantine approximation on abelian varieties, Ann. of Math. (2) 133 (1991), no. 3, 549-576. MR1109353 (93d:11066)

6. William Fulton, Introduction to toric varieties, Annals of Mathematics Studies, vol. 131, Princeton University Press, Princeton, NJ, 1993, The William H. Roever Lectures in Geometry. MR1234037 (94g:14028)

7. Robin Hartshorne, Algebraic geometry, Graduate Texts in Mathematics, No. 52, SpringerVerlag, New York, 1977. MR0463157 (57:3116)

8. Marc Hindry and Joseph H. Silverman, Diophantine geometry, An introduction, Graduate Texts in Mathematics, vol. 201, Springer-Verlag, New York, 2000. MR.1745599 (2001e:11058)

9. Serge Lang, Fundamentals of Diophantine geometry, Springer-Verlag, New York, 1983. MR715605 (85j:11005)

10. David McKinnon, Vojta's main conjecture for blowup surfaces, Proc. Amer. Math. Soc. 131 (2003), no. 1, 1-12 (electronic). MR1929015 (2003g:11071)

11. Charles F. Osgood, A number theoretic-differential equations approach to generalizing Nevanlinna theory, Indiana J. Math. 23 (1981), no. 1-3, 1-15. MR722894 (85b:30043)

12. Hans Peter Schlickewei, Linearformen mit algebraischen koeffizienten, Manuscripta Math. 18 (1976), no. 2, 147-185. MR0401665 (53:5492)

13. Wolfgang M. Schmidt, Linear forms with algebraic coefficients. I, J. Number Theory 3 (1971), 253-277. MR0308061 (46:7176)

14. Joseph H. Silverman, Generalized greatest common divisors, divisibility sequences, and Vojta's conjecture for blowups, Monatsh. Math. 145 (2005), no. 4, 333-350. MR2162351 (2006e:11087)

15. Paul Vojta, Diophantine approximations and value distribution theory, Lecture Notes in Mathematics, vol. 1239, Springer-Verlag, Berlin, 1987. MR883451 (91k:11049) 
16. Yu Yasufuku, Vojta's conjecture on blowups of $\mathbb{P}^{n}, G C D$ inequalities, and the abc conjecture, Monatsh. Math. 163 (2011), no. 2, 237-247. MR2794199

17. _ Vojta's conjecutre and blowups, Ph.D. Dissertation at Brown University.

Department of Mathematics, CUnY-Graduate Center, 365 Fifth Avenue, New York, New York 10016

E-mail address: yasufuku@post.harvard.edu

Current address: Department of Mathematics, Nihon University, 1-8-14 Kanda-Surugadai, Tokyo 101-8308, Japan

E-mail address: yasufuku@math.cst.nihon-u.ac.jp 